\title{
Usefulness of Gram stain examination of peritoneal fluid in postoperative peritonitis to guide empirical antibiotherapy
}

\author{
Pascal Augustin $^{1}$ - Alexy Tran-Dinh ${ }^{1,2} \cdot$ Mathieu Desmard $^{3}$. Sébastien Tanaka ${ }^{1,4} \cdot$ Nathalie Grall $^{5}$. \\ Mouna Ben-Rehouma ${ }^{1} \cdot$ Konstantinos Arapis $^{6} \cdot$ Lara Ribeiro-Parenti $^{6} \cdot$ Philippe Montravers ${ }^{1,7}$
}

Received: 22 March 2019 / Accepted: 22 May 2019 / Published online: 29 May 2019

○) Springer-Verlag GmbH Germany, part of Springer Nature 2019

\begin{abstract}
Purpose In postoperative peritonitis, Gram stain examination (GSE) of peritoneal fluid has been proposed as a guide for the prescription of glycopeptides and antifungal therapy in empirical antibiotherapy. No data support this approach for Grampositive cocci. We aimed to evaluate the performance of GSE in predicting the results of the culture of peritoneal fluid.

Methods In this retrospective single-center study, concordance between GSE and culture of peritoneal fluid was assessed for different types of microorganisms. Factors associated with concordance of the two tests were evaluated in the subpopulation of Gram-positive cocci peritonitis.

Results Among the 152 episodes, the GSE was negative in 57 cases. The negative predictive value and the positive predictive value were $41 \%$ and $87 \%$ for Gram-positive cocci (GPC), $31 \%$ and $86 \%$ for Gram-negative bacilli, and $78 \%$ and $94 \%$ for fungi. GSE is not a reliable guide for the choice of empirical antibiotherapy and cannot reliably rule out the presence of GPC at culture. If we aim to achieve a high rate of adequacy, the systematic use of glycopeptide in the empirical antibiotherapy may be considered.
\end{abstract}

Conclusion GSE shows poor performance to predict the results of culture of peritoneal fluid in postoperative peritonitis. Avoiding covering resistant GPC cannot be based on the result of GSE.

Keywords Gram stain examination · Postoperative peritonitis

Pascal Augustin

pascalaugustin@hotmail.com

1 Département d'Anesthésie Réanimation Chirurgicale et, Groupe Hospitalier Bichat Claude Bernard, Assistance Publique - Hôpitaux de Paris, Université Paris Diderot Sorbonne Paris Cité, 46 rue Henri Huchard, 75018 Paris, France

2 INSERM UMR 1148, Paris, France

3 Service Anesthésie-Réanimation, Centre Hospitalier Sud Francilien, 40 avenue Serge Dassault, 91100 Corbeil-Essones, France

4 INSERM UMR 1188, La Réunion, France

5 Service de Bactériologie, Groupe Hospitalier Bichat Claude Bernard, 46 rue Henri Huchard, 75018 Paris, France

6 Service de Chirurgie Générale, Groupe Hospitalier Bichat Claude Bernard, Assistance Publique - Hôpitaux de Paris, Université Paris Diderot Sorbonne Paris Cité, Paris, France

7 INSERM UMR 1152, Paris, France

\section{Introduction}

Postoperative peritonitis (PP) is a life-threatening complication of abdominal surgery. Compared to communityacquired peritonitis, $\mathrm{PP}$ is associated with a high rate of multidrug-resistant (MDR) bacteria [1, 2]. All bacteria found at culture should be treated since reoperation [3-6]. It has been shown that inadequate empirical antibiotherapy (EA) was associated with unfavorable outcome [1,7]. However, there is no reliable criterion to guide the choice of EA. In the setting of peritonitis, it is recommended to cover Gramnegative bacilli (GNB) and anaerobes. All proposed EA for PP also target beta-lactam-sensitive GPC [5, 6]. To achieve a high adequacy rate, it is proposed to target resistant GPC when risk factors for MDR bacteria are present [8]. Eligible antibiotics in this case are glycopeptides and tigecycline [5, 6].

Some authors and guidelines, including those published by IDSA, suggest guiding the prescription of antibiotics against resistant GPC by the presence of risk factors for 
colonization, a known previous colonization, or the result of Gram stain examination (GSE) of peritoneal fluid [6]. On the contrary, the last French guidelines indicate that GSE is not reliable enough to be used as a guide for the choice of EA. In fact, there is no evidence that GSE can reliably predict the presence of GPC, especially in the setting of PP, in which patients have been previously exposed to antibiotics. Previous antibiotic therapy is known to potentially alter the performance of GSE in other settings, such as in pneumonia [9]. Conversely, no study confirmed the lack of consistency of this approach. We focused on GPC because the result of GSE has no influence on the treatment of GNB, which are systematically targeted. The role of GSE for fungi in peritonitis has already been evaluated in many studies.

No data exists on the performance of the GSE of peritoneal fluid as a guide for EA for coverage of GPC. The goal of the study was to test the ability of GSE to predict the presence of GPC at culture. For the purpose of comparison, we also evaluated the performance of GSE to predict the presence of GNB and fungi. The secondary goal was to identify factors potentially influencing the performance of GSE in predicting the presence of GPC.

\section{Materials and methods}

\section{Study population}

From January 2008 through December 2011, all adult patients with PP requiring admission to the intensive care unit (ICU) were included prospectively in a database and their medical charts were reviewed retrospectively. Postoperative peritonitis was defined as peritoneal infection after abdominal surgery, confirmed by macroscopic findings and peritoneal fluid culture on reoperation yielding at least one colony. In accordance with French law, because this observational study did not modify the physicians' laboratory or clinical practices, the patients or relatives were simply informed about this study; informed consent was not required. The Paris North Hospitals, Paris 7 University, AP-HP Institutional Review Board (IRB), reviewed and approved the study.

\section{Protocol management}

Patients underwent the initial abdominal surgery in our hospital or another institution. They received prophylactic or curative antibiotic therapy in the presence of abdominal infection. All patients had been admitted to the ICU because of organ dysfunction, and all had received standard intensive care management.

\section{Susceptibility testing and empirical antimicrobial therapy}

Peritoneal fluid samples were collected systematically during reoperation and sent immediately to the bacteriology laboratory. The laboratory provides a round-the-clock GSE for peritoneal fluid. The results of GSE and cultures of peritoneal fluid were collected for bacteria and fungi in all PP. Cultures were performed with identification and susceptibility testing for Gram-positive and Gram-negative bacteria, and fungi. Antibiotic susceptibility was determined by the disk diffusion method, according to the criteria of the Antibiotic Susceptibility Testing Committee of the Société Française de Microbiologie [10]. Parenteral EA was started at the time of reoperation according to the recommendations of our institutional protocol. The adjunction of vancomycin was considered in cases of prolonged hospital stay or methicillin-resistant Staphylococcus or amoxicillin-resistant Enterococcus carriage.

\section{Data collection}

Only the first episode of PP was considered for analysis. For comparison, the following information on each patient was collected after admission to the ICU: age; sex; medical past history; previous re operation; characteristics of initial surgery: type, route, and Altemeier wound classification of [11]; duration of antibiotic prescribed at initial surgery; interval antibiotic therapy defined as the administration of antibiotics between initial surgery and reoperation; interval antibiotic therapy and broad-spectrum interval antibiotic therapy; the simplified acute physiology score (SAPS II), and the Sequential Organ Failure Assessment (SOFA) score at the time of reoperation $[12,13]$; medical condition at reoperation; time to reoperation; results of GSE, identification of pathogens in peritoneal fluid; and results of antimicrobial susceptibility tests.

\section{Definitions}

Immunosuppression was defined as current immunosuppressive therapy, corticoid therapy, or uncured cancer. Interval antibiotics (IA) related to any antibiotherapy prescribed between initial surgery and reoperation. Broad-spectrum IA comprised piperacillin/tazobactam, imipenem/cilastatin, cefotaxime, or ceftazidime. Antibiotherapy at initial surgery was the prophylactic or curative antibiotherapy prescribed at the time of initial surgery. Interval reoperation was any reoperation performed before the admission in ICU. Gram stain examination was classified according to its findings: negative GSE; GSE positive for GNB; GSE positive for GPC. 
Resistant GPC were defined as GPC resistant to beta-lactam: amoxicillin-resistant enterococci and methicillin-resistant staphylococci [methicillin-resistant Staphylococcus aureus, coagulase-negative staphylococcus (CNS)]. For each type of bacteria, culture and GSE were defined as concordant, if both were positive, or both were negative. The concordance rate between GSE and culture for one type of bacteria was calculated as follows: numbers of concordant case/total number of PP involving this type of bacteria.

\section{Statistical analysis}

Categorical variables were expressed as numbers and percentages. Continuous variables were expressed as means and percentages. The performance of GSE in predicting the presence of GNB, GPC, and fungi was evaluated by calculating sensitivity, specificity, negative predictive value (NPV), positive predictive value (PPV), and positive and negative likelihood ratio. Categorical variables were compared using a Chi square or exact Fisher test as appropriate, and continuous variables were compared using $t$ test or Mann-Whitney $U$ test as appropriate. Factors associated with concordance between GSE and culture were determined by univariate analysis for PP with GPC. The significance level was 0.05. Statistical analyses were performed with $\mathrm{R}$ software 3.4.2 (The R Foundation for Statistical Computing, Vienna, Austria).

\section{Results}

During the study periods, 152 patients were included in the study. Figure 1 shows the distribution of the population according to the results of the GSE results. Among the 152 episodes, 71 were positive for GPC, 95 for GNB, and 57 were negative at GSE. There were 16 GSE positive for fungi. Tables 1 and 2 show the types of GPC and GNB found at culture according to the results of GSE. The performance of

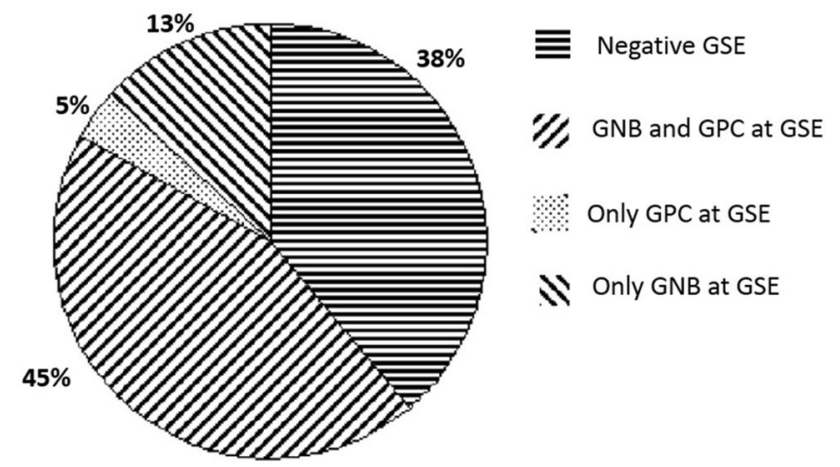

Fig. 1 Distribution of the population according to the results of the GSE results
Table 1 Distribution of different types of GPC according to the results of GSE

\begin{tabular}{lll}
\hline & $\begin{array}{l}\text { Gram-positive } \\
\text { cocci at GSE (71 } \\
\text { PP) }\end{array}$ & $\begin{array}{l}\text { No Gram-positive } \\
\text { cocci at GSE (81 PP) }\end{array}$ \\
\hline Negative culture for GPC & $9(13)$ & $33(41)$ \\
Positive culture for GPC & $62(87)$ & $48(59)$ \\
Streptococcus sp. & $24(34)$ & $7(9)$ \\
Staphylococcus $\mathrm{sp}$. & $22(31)$ & $20(25)$ \\
Enterococcus $\mathrm{sp}$. & $33(46)$ & $27(33)$ \\
Resistant GPC & $18(25)$ & $25(31)$ \\
Staphylococcus $\mathrm{sp}$. & $12(17)$ & $20(25)$ \\
Enterococcus $\mathrm{sp}$. & $6(8)$ & $6(7)$ \\
\hline
\end{tabular}

GSE Gram stain examination, $P P$ postoperative peritonitis, GPC Gram-positive cocci

GSE to predict the results of cultures of peritoneal fluid is provided in Table 3. The GSE demonstrated a poor negative predictive value for GNB, GPC, and fungi. Not surprisingly, the performance of GSE for resistant GPC was also weak. Table 4 provides the ratio of negative and positive GSE for each type of bacteria. The concordance rate clearly varied according to the type of bacteria $(p=0.015)$.

Analysis performed in the subpopulation of GPC PP showed that factors associated with concordance between GSE and culture for GPC were the duration of the antibiotherapy prescribed for the initial surgery, an ongoing antibiotherapy at the time of reoperation, the delay of reoperation, and the cause of PP (Table 5).

\section{Discussion}

The GSE of peritoneal fluid does not reliably predict the results of culture in the setting of PP. Therefore, if we aim to optimize the rate of adequate EA for PP, GSE cannot be used for guiding antibiotherapy.

Table 2 Distribution of different types of GNB according to the results of GSE

\begin{tabular}{lll}
\hline & $\begin{array}{l}\text { Gram-negative } \\
\text { bacilli at GSE (84 } \\
\text { PP) }\end{array}$ & $\begin{array}{l}\text { No Gram-negative } \\
\text { bacilli at GSE (68 } \\
\text { PP) }\end{array}$ \\
\hline Negative culture for GNB & $12(14)$ & $21(31)$ \\
Positive culture for GNB & $62(74)$ & $57(84)$ \\
Enterobacteriaceae & $71(85)$ & $51(75)$ \\
Non-fermenting bacilli & $12(14)$ & $11(16)$ \\
EB and NFB & $11(13)$ & $5(7)$ \\
\hline
\end{tabular}

GNB Gram-negative bacilli, GSE Gram stain examination, EB enterobacteriaceae, NFB non-fermenting 
Table 3 Performance of GSE in predicting the results of peritoneal fluid culture for GPC, RGPC, and GNB bacilli

\begin{tabular}{lllllll}
\hline & Sensitivity & Specificity & PPV & NPV & LR + & LR - \\
\hline All GPC & $56(61 / 109)$ & $77(33 / 43)$ & $86(61 / 71)$ & $41(33 / 81)$ & 2.4 & 0.6 \\
RGPC & $40(17 / 42)$ & $51(56 / 110)$ & $24(17 / 71)$ & $69(56 / 81)$ & 0.8 & 1.2 \\
GNB & $61(72 / 119)$ & $64(21 / 33)$ & $86(72 / 84)$ & $31(21 / 68)$ & 1.7 & 0.6 \\
Fungi & $35(16 / 46)$ & $99(105 / 106)$ & $94(16 / 17)$ & $78(105 / 135)$ & 35 & 0.7 \\
\hline
\end{tabular}

$P P V$ positive predictive value, $N P V$ negative predictive value, $L R$ likelihood ratio, $C I$ confidence interval, $G P C$ Gram-positive cocci, GNB Gram-negative bacilli, $R G P C$ resistant Gram-positive cocci

Table 4 Ratio of negative and positive GSE for each type of bacteria found at culture of peritoneal fluid

\begin{tabular}{lllllllll}
\hline & Strepto sp. $(n=31)$ & Entero sp. $(n=60)$ & $\begin{array}{l}\text { Staphylo sp. } \\
(n=43)\end{array}$ & $\begin{array}{l}\text { R entero sp. } \\
(n=13)\end{array}$ & $\begin{array}{l}\text { R Staphylo } \\
\text { sp. }(n=32)\end{array}$ & EB $(n=111)$ & NFB $(n=23)$ & $p$ \\
\hline Neg GSE & $7(23)$ & $27(45)$ & $21(49)$ & $4(31)$ & $21(66)$ & $40(36)$ & $11(48)$ \\
Pos GSE & $24(77)$ & $33(55)$ & $22(51)$ & $9(69)$ & $11(34)$ & $71(64)$ & $12(52)$ & 0.015 \\
\hline
\end{tabular}

Strepto Streptococcus, Entero Enterococcus, Staphylo Staphylococcus, $R$ Strepto resistant Streptococcus, $R$ Entero resistant Enterococcus, $R$ Staphylo resistant Staphylococcus, EB Enterobacteriaceae, NFB non-fermenting bacilli, NegGSE negative Gram stain examination, Pos GSE positive Gram stain examination

We have observed that the performance of GSE in predicting the result of cultures varies according to the type of microorganism. The poor performance of GSE to guide the coverage of resistant GPC is not surprising. Nevertheless, some current guidelines still advocate the use of Gram stain examination to guide the prescription of glycopeptide. We do need studies to validate or refute this practice. This is the only study providing data regarding this issue.

It is proposed to base the prescription of glycopeptides on the GSE, and on the presence of risk factors for multidrug resistant bacteria $[5,6]$. Some studies have explored the risk factors for MDR bacteria [14, 15], but no studies have specifically addressed the issue of predicting the presence of resistant GPC.

There are two reasons that may encourage restraining the use of glycopeptide. Besides the impact on renal function [16], it has been shown that glycopeptide administration was associated with an increased MIC of bacteria responsible for subsequent infections [17]. The administration of glycopeptide is not consensual, but GSE is still used to guide EA, including in PP, in everyday practice [18]. There is no published data indicating when the coverage of resistant GPC may be safely avoided [19].

All bacteria including resistant GPC in PP have to be treated [20,21], but some specific issues are still debated [22]. The timing of initiation of glycopeptides has never been specifically addressed. Because of their lower pathogenicity, we may consider treating CNS and énterococci after receiving the result of peritoneal fluid culture. This specific approach has not been investigated and would contradict the need for prompt adequate EA.

In our study, we found some potential factors decreasing the performance of GSE in predicting GPC at culture. Some non-evaluated factors, like the training of the doctor performing the GSE, or night shift timings may also affect the results of GSE. In the setting of ventilator-associated pneumonia (VAP), the sensitivity of GSE varies widely with a pooled sensitivity of GSE for VAP of 79\% [23].

Some limitations exist. This is a single-center retrospective study. The result of GSE is highly operator dependent. Some institutions do not perform immediate GSE or cannot perform it during night shift hours. Some factors affecting the performance of GSE may not have been recorded. The role of anaerobes which could explain the poor performance of GSE has not been explored. This study is purely microbiological and was conducted with no relation to the clinical outcome.

\section{Conclusion}

Gram stain examination of peritoneal fluid is of poor performance in predicting the results of culture. Therefore, it cannot guide the prescription of glycopeptides when treating patients with PP. 
Table 5 Characteristics of patients with GPC at culture: comparison of episodes with concordant and non-concordant GSE

\begin{tabular}{|c|c|c|c|}
\hline & $\begin{array}{l}\text { No concord- } \\
\text { ance }(n=48)\end{array}$ & Concordance $(n=61)$ & \\
\hline \multicolumn{4}{|l|}{ General characteristics } \\
\hline Gender (male) & $31(65)$ & $32(52)$ & 0.20 \\
\hline Age (years) & $62 \pm 18$ & $59 \pm 16$ & 0.28 \\
\hline Immunosuppression & $16(33)$ & $25(41)$ & 0.41 \\
\hline Diabetes mellitus & $8(17)$ & $9(15)$ & 0.78 \\
\hline Antibiotherapy 3 months prior to initial surgery & $9(19)$ & $20(33)$ & 0.10 \\
\hline Hospitalization 3 months prior to initial surgery & $16(33)$ & $30(49)$ & 0.10 \\
\hline \multicolumn{4}{|l|}{ Initial surgery } \\
\hline Upper gastrointestinal tract for initial surgery & $24(50)$ & $25(41)$ & 0.35 \\
\hline Septic or contaminated initial surgery & $24(50)$ & $22(36)$ & 0.14 \\
\hline Emergent initial surgery & $23(48)$ & $21(34)$ & 0.15 \\
\hline Duration of initial antibiotherapy (days) & $2(1-4)$ & $1(1-2)$ & 0.048 \\
\hline Interval reoperation & $13(27)$ & $15(25)$ & 0.77 \\
\hline Interval antibiotherapy & $34(71)$ & $34(56)$ & 0.11 \\
\hline Broad-spectrum interval antibiotherapy & $21(44)$ & $19(31)$ & 0.18 \\
\hline \multicolumn{4}{|l|}{ Reoperation } \\
\hline Current antibiotherapy at the time of reoperation & $33(69)$ & $29(48)$ & 0.026 \\
\hline Time since initiation of current antibiotherapy (days) & $2(0-4)$ & $0(0-5)$ & 0.33 \\
\hline Time to reoperation (days) & $7(4-9)$ & $9(6-17)$ & 0.002 \\
\hline SAPS 2 on admission in ICU & $48 \pm 15$ & $45 \pm 16$ & 0.37 \\
\hline SOFA on admission in ICU & $7 \pm 4$ & $7 \pm 4$ & 0.67 \\
\hline White blood cell count $\left(10^{9} / \mathrm{L}\right)$ & $17.1 \pm 12$ & $19.1 \pm 12$ & 0.33 \\
\hline \multicolumn{4}{|l|}{ Source of PP } \\
\hline Upper gastrointestinal tract cause & $20(42)$ & $22(36)$ & 0.37 \\
\hline Non-upper gastrointestinal tract cause & $21(44)$ & $34(56)$ & \\
\hline No cause found & $7(15)$ & $5(8)$ & \\
\hline \multicolumn{4}{|l|}{ Cause of PP } \\
\hline Bowel perforation & $8(17)$ & $27(44)$ & 0.018 \\
\hline Anastomosis leakage & $19(40)$ & $18(30)$ & \\
\hline Other cause & $14(29)$ & $11(18)$ & \\
\hline No cause found & $7(15)$ & $5(8)$ & \\
\hline
\end{tabular}

Categorical variables expressed as number (percentage), normally distributed variables expressed as mean \pm standard deviation, and non-normally distributed variables expressed as median (IQR)

$P P$ postoperative peritonitis

\section{Compliance with ethical standards}

Conflict of interest Pascal Augustin, Alexy Tran-Dinh, Mathieu Desmard, Sébastien Tanaka, Nathalie Grall, Mouna Ben-Rehouma, Konstantinos Arapis, Lara Ribeiro-Parenti, and Philippe Montravers declare that they have no conflict of interest.

Research involving human participants Authorization of the institutional review board was obtained.

\section{References}

1. Montravers P, Gauzit R, Muller C, Marmuse JP, Fichelle A, Desmonts JM. Emergence of antibiotic-resistant bacteria in cases of peritonitis after intraabdominal surgery affects the efficacy of empirical antimicrobial therapy. Clin Infect Dis. 1996;23:486-94.

2. Montravers P, Dufour G, Guglielminotti J, Desmard M, Muller C, Houissa H, Allou N. Dynamic changes of microbial flora and therapeutic consequences in persistent peritonitis. Crit Care. 2015;19:70.

3. Sartelli M, Catena F, Abu-Zidan FM, Ansaloni L, Biffl WL, Boermeester MA, et al. Management of intra-abdominal infections: recommendations by the WSES 2016 consensus conference. World J Emerg Surg. 2017;12:22.

4. Mazuski JE, Tessier JM, May AK, Sawyer RG, Nadler EP, Rosengart MR, et al. The surgical infection society revised guidelines on the management of intra-abdominal infection. Surg Infect (Larchmt). 2017;18:1-76.

5. Montravers P, Dupont H, Leone M, Constantin JM, Mertes PM, Société française d'anesthésie et de réanimation (Sfar); Société de réanimation de langue française (SRLF), Laterre PF, Misset B, Société de pathologie infectieuse de langue française (SPILF), Bru 
JP, Gauzit R, Sotto A, Association française de chirurgie (AFC), Brigand C, Hamy A, Société française de chirurgie digestive (SFCD), Tuech JJ. Guidelines for management of intra-abdominal infections. Anaesth Crit Care Pain Med. 2015;34:117-30.

6. Solomkin JS, Mazuski JE, Bradley JS, Rodvold KA, Goldstein EJ, Baron EJ, O'Neill PJ, Chow AW, Dellinger EP, Eachempati SR, Gorbach S, Hilfiker M, May AK, Nathens AB, Sawyer RG, Bartlett JG. Diagnosis and management of complicated intra-abdominal infection in adults and children: guidelines by the Surgical Infection Society and the Infectious Diseases Society of America. Clin Infect Dis. 2010;50(2):133-64.

7. Krobot K, Yin D, Zhang Q, Sen S, Altendorf-Hofmann A, Scheele J, Sendt W. Effect of inappropriate initial empiric antibiotic therapy on outcome of patients with community-acquired intra-abdominal infections requiring surgery. Eur J Clin Microbiol Infect Dis. 2004;23(9):682-7.

8. Steinbach CL, Töpper C, Adam T, Kees MG. Spectrum adequacy of antibiotic regimens for secondary peritonitis: a retrospective analysis in intermediate and intensive care unit patients. Ann Clin Microbiol Antimicrob. 2015;14:48.

9. Musher DM, Montoya R, Wanahita A. Diagnostic value of microscopic examination of Gram-stained sputum and sputum cultures in patients with bacteremic pneumococcal pneumonia. Clin Infect Dis. 2004;39(2):165-9.

10. Members of the SFM Antibiogram Committee. Comité de l'Antibiogramme de la Société Française de Microbiologie report 2003. Int J Antimicrob Agents. 2003;21(4):364-91.

11. Altemeier WA, Culbertson WR, Hummel RP. Surgical considerations of endogenous infections-sources, types, and methods of control. Surg Clin North Am. 1968;48:227-40.

12. Vincent JL, Moreno R, Takala J, Willatts S, De Mendonça A, Bruining H, Reinhart CK, Suter PM, Thijs LG. The SOFA (Sepsisrelated organ failure assessment) score to describe organ dysfunction/failure. On behalf of the Working Group on Sepsis-Related Problems of the European Society of Intensive Care Medicine. Intensive Care Med. 1996;22(7):707-10.

13. Le Gall JR, Lemeshow S, Saulnier F. A new simplified acute physiology score (SAPS II) based on a European/North American multicenter study. JAMA. 1993;270(24):2957-63.
14. Augustin P, Kermarrec N, Muller-Serieys C, Lasocki S, Chosidow D, Marmuse JP, Valin N, Desmonts JM, Montravers P. Risk factors for multidrug resistant bacteria and optimization of empirical antibiotic therapy in postoperative peritonitis. Crit Care. 2010;14(1):R20.

15. Seguin P, Fédun Y, Laviolle B, Nesseler N, Donnio PY, Mallédant Y. Risk factors for multidrug-resistant bacteria in patients with post-operative peritonitis requiring intensive care. J Antimicrob Chemother. 2010;65(2):342-6.

16. Filippone EJ, Kraft WK, Farber JL. The nephrotoxicity of vancomycin. Clin Pharmacol Ther. 2017;102(3):459-69.

17. Moise PA, Smyth DS, El-Fawal N, Robinson DA, Holden PN, Forrest A, Sakoulas G. Microbiological effects of prior vancomycin use in patients with methicillin-resistant Staphylococcus aureus bacteraemia. J Antimicrob Chemother. 2008;61(1):85-90.

18. Montravers P, Dupont H, Gauzit R, Veber B, Bedos JP, Lepape A, Club d'Infectiologie en Anesthésie-Réanimation Study Group. Strategies of initiation and streamlining of antibiotic therapy in 41 French intensive care units. Crit Care. 2011;15(1):R17.

19. Tarchini G. Empirical enterococcal coverage for complicated intra-abdominal infection. Clin Infect Dis. 2010;51(6):757-8.

20. Sitges-Serra A, Lòpez MJ, Girvent M, Almirall S, Sancho JJ. Postoperative enterococcal infection after treatment of complicated intra-abdominal sepsis. Br J Surg. 2002;89:361-7.

21. Burnett RJ, Haverstock DC, Dellinger EP, Reinhart HH, Bohnen JM, Rotstein OD, Vogel SB, Solomkin JS. Definition of the role of enterococcus in intraabdominal infection: analysis of a prospective randomized trial. Surgery. 1995;118(4):716-21.

22. Montravers P, Tashk P, Tran Dinh A. Unmet needs in the management of intra-abdominal infections. Expert Rev Anti Infect Ther. 2017;15:839-50.

23. O'Horo JC, Thompson D, Safdar N. Is the gram stain useful in the microbiologic diagnosis of VAP? A meta-analysis. Clin Infect Dis. 2012;55(4):551-61. 